\title{
O ensino do exame de fundo de olho: vivências e percepções de estudantes de medicina
}

The teaching of ocular fundus exam: experiences and perceptions of medicine students

\author{
Norimar Pinto de Oliveira' ${ }^{1}$ (D) norimar.oliveira@terra.com.br \\ Robson Jose de Souza Domingues' (1) domingues@uepa.br \\ Edmundo Frota Almeida de Almeida Sobrinho' (1) edmundofalmeida@gmail.com \\ Simone Argentino' 1 (1) simone.argentino@uepa.br \\ Ellen Ágatta Marinho Silva' (1) ellenagatta.marinho@gmail.com \\ Marcelo Dias Ferreira Júnior' ${ }^{1}$ (D) marcelojunio1997@gmail.com
}

\section{RESUMO}

Introdução: O ensino médico vem passando por transformações nas últimas décadas. Objetivos educacionais tendem a se alterar com os avanços tecnológicos recentes, em especial na área de tecnologias de informação.

Objetivo: Esta pesquisa aborda o exame do fundo de olho explorando e analisando as dificuldades dos estudantes de Medicina na execução desse componente do exame clínico e busca propor diretrizes para seu ensino na graduação médica.

Métodos: Trata-se de uma pesquisa qualitativa com técnicas de observação direta e entrevistas com análise de conteúdo em uma população de estudantes do internato da Universidade do Estado do Pará (Uepa), na cidade de Marabá. Na avaliação de conteúdo utilizaram-se recursos do programa livre de análise de texto Iramuteq.

Resultados: Dos 21 estudantes voluntários participantes da pesquisa, apenas dois relataram experiência anterior com oftalmoscópio direto (9,52\%) e um aluno havia participado de campanha com uso de dispositivo portátil para registro da imagem do fundo de olho (4,8\%). As atividades da pesquisa incluiram discussão de casos clínicos, realização de oftalmoscopias diretas em pacientes voluntários e análise de retinografias. Na análise dos textos correspondentes às entrevistas foram categorizadas quatro classes geradas pelo programa Iramuteq, realçando-se o valor da integração de teoria e prática no depoimento dos alunos.

Conclusão: Programas de treinamento com integração de teoria e prática e valendo-se de princípios de aprendizagem significativa podem contribuir para prover competência ao estudante de Medicina para o exame de fundo de olho, adequando-se ao surgimento de novas tecnologias.

Palavras-chave: Fundo de Olho; Educação Médica; Aprendizagem Baseada em Problemas; Segmento Posterior do Olho; Ensino.

\section{ABSTRACT}

Introduction: Medical education has undergone changes in recent decades. Educational objectives tend to change with recent technological advances, especially in the area of information technologies.

Objective: This research addresses the examination of ocular fundus by exploring and analyzing the difficulties medical students encounter in relation to this component of the clinical examination and seeks to propose guidelines for its teaching in undergraduate medical training.

Method: Qualitative research with direct observation techniques and interviews with content analysis with a population of students at the University of the State of Pará (UEPA), in the city of Marabá. Content analysis used resources from the free text analysis program Iramuteq.

Results: Of the 21 volunteer students participating in the research, only two reported previous experience with direct ophthalmoscope (9.52\%) and one student had participated in a campaign using a portable device to record the fundus image (4.8\%). Research activities included discussion of clinical cases, performing direct ophthalmoscopies in volunteer patients and retinography analysis. In the analysis of the texts corresponding to the interviews, four classes generated by the Iramuteq program were categorized, highlighting the value of theoretical and practical integration in the students' testimony.

Conclusion: Training programs with theoretical and practical integration using meaningful learning concepts can help qualify medical students in the ocular fundus exam, adapting to the emergence of new technologies.

Keywords: Eye Fundus; Medical Education; Problem-Based Learning; Posterior Segment of the Eye; Teaching.

${ }^{1}$ Universidade do Estado do Pará, Belém, Pará, Brasil.

Editora-chefe: Rosiane Viana Zuza Diniz

Editor associado: Kristopherson Lustosa Augusto

Recebido em 04/10/20; Aceito em 05/04/21.

Avaliado pelo processo de double blind review. 


\section{INTRODUÇÃO}

O ensino do exame de fundo de olho na graduação médica vem sendo um tema recorrente na literatura e, muitas vezes, controverso. Estudos assinalam a pouca oferta de conteúdos relacionados à oftalmologia aos estudantes de Medicina e a pouca familiaridade no uso do oftalmoscópio direto ou de outras técnicas para análise do fundo de olho por médicos não oftalmologistas ${ }^{1-12}$. A tendência atual aponta para a avaliação do fundo de olho por meio da obtenção de imagens de alta definição com aparelhos portáteis de fácil manuseio em substituição ao tradicional uso do oftalmoscópio direto, em decorrência dos avanços tecnológicos dos últimos anos e das limitações da técnica tradicional 1,6,13-21.

O sistema neurovisual (olho - pares de nervos cranianos - cérebro) e suas relações com o organismo humano como um todo associam-se com frequência com complicações decorrentes de agravos de alta prevalência, como diabetes melittus (DM), hipertensão arterial sistêmica (HAS) e síndrome metabólica (SM); outras condições afetam a visão, como tumores do sistema nervoso central, síndrome da hipertensão intracraniana, doenças neurodegenerativas, distúrbios reumáticos, hematológicos e infecciosos. Alterações do funcionamento do sistema neurovisual podem configurar situações de urgência e sua avaliação é necessária para a tomada de decisões que podem ser críticas para os pacientes. O exame do aparelho ocular e de sua função visual e o exame do fundo de olho permitem o acesso a dados clínicos que caracterizam esse sistema como um "sistema-alvo" ou então, em outras ocasiões, um "sistema sentinela", apresentando sinais e definindo sintomas que devem ser conhecidos por um profissional médico generalista ${ }^{10,12,22-25}$.

Muitos trabalhos sobre o ensino do exame de fundo de olho utilizam modelos de simulação (como uso de bonecos e dispositivos virtuais), imagens padronizadas de fundo de olho, jogos interativos e aplicativos com a intenção de otimizar o processo de aprendizagem desse exame ${ }^{26-35}$. A pluralidade e o valor desses diversos recursos usados em uma lógica da integração de teoria e prática aliados a uma pedagogia adequada podem potencializar a aquisição de competência para a realização do exame de fundo de olho. Estudos sobre temas oftalmológicos realizados em universidades brasileiras que utilizaram princípios inovadores de aprendizagem foram conduzidos por pesquisadores brasileiros com resultados interessantes ${ }^{36}$.

O ensino médico vem passando por modificações progressivas nas últimas décadas. Novos paradigmas estão sendo constituídos após um período de incentivos no sentido da especialização precoce e de uma valorização excessiva de tecnologias e procedimentos de alta complexidade. $\mathrm{O}$ ensino médico se modifica à medida que novas tecnologias nos campos do diagnóstico, da prevenção primária e secundária e da terapêutica clínica e cirúrgica vão se incorporando aos serviços de saúde. Tecnologias de informação, modelos de simulação para treinamento e, recentemente, programas de inteligência artificial $(\mathrm{IA})^{37-39}$ despontam e se afirmam nos ambientes dos serviços e das ações de saúde, em educação continuada e também no ensino da graduação médica.

Em relação ao ensino específico da técnica tradicional da oftalmoscopia direta para o exame de fundo de olho é importante sublinhar divergências sobre o tema ${ }^{10-11}$. Um debate recente foi estabelecido sobre a pertinência ou não de se utilizar o oftalmoscópio direto, dadas as suas limitações (em comparação com outras técnicas) e a facilidade e disponibilidades crescentes da obtenção de imagens de alta resolução do fundo de olho. Técnicas de diagnóstico e recursos de ensino precisam estar adequados aos objetivos educacionais das diferentes fases da formação médica seja para otimizarem a acurácia de médicos residentes, seja para servirem como modelos e padrões para a educação médica nos níveis de graduação ou ainda permitirem aplicações à educação médica continuada. Durante a graduação médica, uma meta geral de ensino plausível deve ser o reconhecimento da necessidade da realização do exame do fundo de olho por meio de técnicas adequadas e seguras, em situações epidemiologicamente relevantes, nas quais a oftalmoscopia constitua elemento importante para as tomadas de decisões referentes ao manejo clínico 3,31,40.

$\mathrm{Na}$ literatura observa-se uma questão central pouco explorada a respeito do ensino do exame de fundo de olho na graduação médica:

- independentemente das técnicas para a execução do exame de fundo de olho, o ensino desse tema vem ocorrendo de forma contextualizada, com efetiva integração de teoria e prática, ou seja, está inserido nos horizontes de integração curricular e de princípios de aprendizagem significativa?

As dificuldades para adquirir e manter competências nessa área podem se relacionar a conceitos e práticas pedagógicas e às estruturas curriculares do ensino médico. Nesse sentido, este estudo teve como objetivos principais detectar essas dificuldades e buscar soluções para que o estudante de Medicina adquira competência para essa etapa da avaliação clínica.

Esta pesquisa não avaliou a acurácia do exame praticado pelos estudantes nem validou novas técnicas de diagnóstico, mas explorou o problema do ensino do exame do fundo de olho de um ponto de vista pedagógico, dada a relevância do seu valor na atenção primária à saúde e em serviços de urgência ou emergência. 


\section{MÉTODOS}

Trata-se de estudo qualitativo que utilizou técnicas de observação direta e entrevistas com análise de conteúdo com suporte do programa Interface de $\mathrm{R}$ pour les Analyses Multidimensionnelles de Textes et de Questionnaires (Iramuteq). O estudo foi conduzido em uma população de estudantes matriculados no quinto e sexto anos do curso de Medicina da Universidade do Estado do Pará (Uepa) convocados por redes sociais. Além do campus, foram locais da pesquisa o ambulatório o setor de retinografia de um serviço conveniado ao Sistema Único de Saúde (SUS). Pacientes voluntários foram atendidos durante a etapa de treinamento de habilidades supervisionado em ambulatório.

A metodologia adotada baseou-se em conceitos e práticas de aprendizagem significativa e de aprendizagem baseada em problemas e foi conduzida em quatro etapas a seguir descritas.

\section{Primeira etapa: Discussão de casos clínicos}

Dividiu-se a população do estudo em dois grupos de alunos: um com dez participantes e outro com 11. Realizaramse apresentação e a discussão participativa de casos clínicos de pacientes hipertensos e diabéticos com lesões em órgãos-alvo. Os objetivos dessa primeira etapa foram sensibilizar e estimular os estudantes para a importância do exame de fundo de olho, em especial na avaliação de grupos populacionais específicos e prover a integração de teoria e prática às atividades, de modo a incentivar o estudo individual antes das atividades práticas.

\section{Segunda etapa: Prática de habilidades em pacientes voluntários}

Os participantes foram divididos em três grupos de cinco alunos e um grupo com seis alunos que realizaram atividades de treino de habilidades em um período de quatro horas e meia / grupo. Treinou-se a técnica de oftalmoscopia direta com o manejo e posicionamento adequado do examinador e do oftalmoscópio direto em relação ao paciente e a visualização das referências do disco ótico e da emergência dos vasos da retina. Esses procedimentos foram realizados sem midríase medicamentosa. Nessas etapas, o pesquisador, médico oftalmologista, esteve sempre presente, orientando e supervisionando os estudantes. Utilizaram-se três oftalmoscópios diretos Welch Allyn modelo 11730 . O treino foi realizado em ambulatório com luzes diminuídas.

\section{Terceira etapa: Visita e interação ao serviço de retinografia}

Os grupos visitaram o setor de retinografia onde puderam observar a obtenção de imagens de alta definição e analisar, com o médico pesquisador, imagens pré-selecionada de modo que pudessem verificar as alterações presentes em retinopatias secundárias à DM e HAS.

\section{Quarta etapa: Devolutiva dos estudantes}

Por meio de entrevistas com questões semiabertas, os estudantes avaliaram e comentaram as atividades realizadas de ensino e pesquisa e puderam oferecer sugestões sobre o processo de aprendizado.

\section{Coleta e análise de dados}

Dados de observação direta foram anotados em fichas pelo médico oftalmologista responsável pela pesquisa. Os discentes foram identificados com códigos E1 a E21. Parte das observações gerais é resumida no Quadro 1.

A coleta de dados completou-se com as devolutivas dos estudantes, na forma das entrevistas individuais que foram gravadas em smartphone e depois transcritas na íntegra para formação do corpus. Análises textuais requerem a criação de um corpus, que representa uma coleção de textos sobre um determinado tema. As informações obtidas pelas entrevistas foram avaliadas por meio da técnica de análise de conteúdo realizada com o suporte do programa livre de análise de texto Iramuteq.

Quadro 1. Síntese das observações de campo.

\section{ETAPA}

DISCUSSÃO DE CASOS CLINICOS

NÍVEL REGULAR

HABILIDADES EM FUNDOSCOPIA DIRETA

NÍVEL BAIXO

CONHECIMENTOS E HABILIDADES PARA ANÁLISE DE RETINOGRAFIAS

\section{AVALIAÇÃO}

Embora tenham bons conhecimentos sobre diagnóstico e tratamento da HAS e DM, os estudantes demonstraram pouco conhecimento sobre conceitos importantes como "órgão-alvo" e a natureza anatômica e fisiológica da alterações vasculares causadas por esses agravos.

Nenhum estudante possuía habilidade para manipular o oftalmoscópio direto e todos apresentaram dificuldades de conseguir focalizar o polo posterior.

Houve reconhecimento parcial de alguns sinais de retinopatia NÍVEL REGULAR (como hemorragias e exsudatos) mas os alunos desconheciam o roteiro sistematizado de análise de retinografias.

Fonte: Bloco de anotações de campo do pesquisador. 
A pesquisa obedeceu às normas regulamentadoras de pesquisas que envolvem os seres humanos - Resolução do Conselho Nacional de Saúde (CNS) no 466/2012. Aos participantes da pesquisa - discentes e pacientes voluntários foram apresentados previamente os objetivos do projeto e o Termo de Consentimento Livre e Esclarecido (TCLE). A pesquisa foi aprovada pelo Comitê de Ética em Pesquisa da Uepa: Parecer no 3.530.758 - Certificado de Apresentação para Apreciação Ética (CAAE) no 15058719.5.0000.8607.

\section{RESULTADOS}

Os 21 estudantes participantes da pesquisa corresponderam a $51 \%$ do total de matriculados no internato, $47,6 \%$ eram do sexo feminino e $54,4 \%$ do sexo masculino. A idade variou de 21 anos a 32 anos, com mediana de 24 anos. Quanto ao ano cursado, $52,4 \%$ cursavam o quinto ano e $47,6 \%$ o sexto. Após a realização das discussões de casos clínicos (duração de uma hora e meia por grupo), todos os grupos realizaram oftalmoscopias diretas em pacientes voluntários. O nível médio de conhecimentos teóricos dos estudantes foi registrado como regular pelo pesquisador, em especial por conta da dificuldade de compreenderem o componente anatômico e funcional de alterações vasculares e do fluxo sanguíneo causadas pela HAS e DM.

Após a leitura do TCLE, 20 pacientes voluntários foram examinados nos quais se observaram diagnósticos sistêmicos e oftalmológicos que não necessariamente se traduziram em exames de fundo de olho alterados. Dois pacientes referiram HAS, com um apresentando no exame de fundo de olho aumento da tortuosidade vascular.

Apenas dois estudantes relataram experiência única e limitada com oftalmoscópio direto. Um deles havia participado de um mutirão de saúde promovido pela Polícia Rodoviária para caminhoneiros e teve contato com a obtenção de imagens de fundo de olho com dispositivo portátil.

Como nenhum estudante sabia manipular o oftalmoscópio o nível de habilidade foi considerado baixo pelo pesquisador (Quadro 1).Todos tiveram de ser orientados quanto ao mecanismo de ligar e desligar o aparelho, à regulação das lentes para focalização do segmento posterior, à necessidade de sala escura ou semiescura, ao posicionamento do paciente e do examinador (pega do aparelho com braço direito para examinar com olho direito o olho direito do paciente e a mesma regra para o lado esquerdo) e à angulação do aparelho em relação à linha média nasal do paciente. Todos os estudantes foram orientados a informar o paciente sobre o ofuscamento e solicitar que qualquer incômodo fosse informado para que o procedimento pudesse ser interrompido. Após algumas tentativas, o que variou de uma a cinco, todos referiram ter conseguido visualizar as estruturas de referência determinadas. No geral todos os estudantes apresentaram dificuldades motoras que foram se dissipando com a repetição dos exames.

Durante o processo de treinamento, os discentes solicitaram permissão para realizar o exame entre pares, o que foi consentido pelo pesquisador.

As retinografias com alterações de retinopatia secundária à HAS e/ou DM foram demonstradas aos alunos em tela de computador e todos os discentes puderam visitar o setor de retinografia. Nos grupos abordaram-se cinco retinografias e demonstrou-se o roteiro básico de interpretação (coloração e aparência do disco ótico, relação escavação - diâmetro total, trajetória e calibre dos vasos, presença de microaneurismas, hemorragias, exsudatos ou áreas de isquemia, visualização e aparência da mácula). O roteiro de interpretação das retinografias era desconhecido pelos estudantes

O tempo despendido com as atividades de habilidades em fundoscopia e avaliação de retinografias totalizou quatro horas e meia por grupo, o que somado à discussão teórica totalizou seis horas de atividades por grupo de cinco ou seis alunos.

Ao final das atividades todas as entrevistas foram gravadas em smartphone e transcritas por programa de voz para texto e as transcrições foram tratadas para a formação do corpus para alimentar o programa Iramuteq.

O corpus geral que alimentou o programa Iramuteq foi constituído por 21 textos, separados em 248 segmentos de texto (ST), com aproveitamento 182 ST (73,39\%).

O conteúdo analisado foi distribuído pelo programa em cinco classes: classe 1, com 42 ST (23,08\%); classe 2, com 34 ST (18,68\%); classe 3, com 34ST (18,68\%); classe 4, com 36ST (19,78\%); classe 5, com 36 ST (19,78\%); em seguida, fez-se a categorização das classes. Como não se encontrou uma significante correlação entre a classe 2 e o contexto geral da pesquisa e das entrevistas resolveu-se por sua exclusão do processo de análise de resultados, permanecendo assim como bases do roteiro as classes que apresentaram correlação mais forte. As tabelas 1,2,3 e 4 demonstram essas classes e as ocorrências das palavras dos textos com suas frequências absoluta e relativa e força de associação (qui-quadrado) com o conteúdo de cada classe.

\section{DISCUSSÃO}

Pelas anotações de observação direta, verificou-se que o nível de conhecimento teórico sobre o tema fundo de olho encontrava-se desnivelado em relação à prática do exame de oftalmoscopia (Quadro 1). Enquanto os estudantes manifestaram um nível regular de conhecimento nas discussões de casos clínicos, eles apresentaram um nível prévio de habilidades baixo para a oftalmoscopia direta que somente foi melhorado com a repetição dos exames em pacientes voluntários. 
Tabela 1. Classe 1: Percepção de ofertas de conteúdo de oftalmologia no curso de Medicina. Palavras e suas frequências por ordem decrescente das que possuem maior associação com a classe, de acordo $\operatorname{com} X^{2}>3,80, p<0,05$.

\begin{tabular}{ccc}
\hline Palavra & $\boldsymbol{f}$ & $\mathbf{X}^{\mathbf{2}}$ \\
\hline Contato & 18 & 41,9 \\
Oftalmologia & 16 & 41,0 \\
Nunca & 7 & 24,2 \\
Caso & 12 & 21,3 \\
Curso & 7 & 19,5 \\
Faculdade & 8 & 19,3 \\
Universidade & 4 & 13,6 \\
Apenas & 7 & 13,1 \\
Parte & 11 & 12,9 \\
Menos & 5 & 12,7 \\
\hline
\end{tabular}

Fonte: Relatório de análise textual processado pelo programa Iramuteq.

Tabela 2. Classe 3: Percepção da retinografia comparada à fundoscopia. Palavras e suas frequências por ordem decrescente das que possuem maior associação com a classe, de acordo com $X^{2}>3,80, p<0,05$.

\begin{tabular}{ccc}
\hline Palavra & $\boldsymbol{f}$ & $\mathbf{X}^{\mathbf{2}}$ \\
\hline Facilitar & 6 & 27,0 \\
Bastante & 8 & 19,5 \\
Detalhe & 4 & 17,8 \\
Retinografia digital & 6 & 17,5 \\
Retina & 6 & 17,5 \\
Visualização & 5 & 17,1 \\
Análise & 8 & 16,9 \\
Saber & 9 & 16,3 \\
Retinografia & 9 & 14,5 \\
Pontos positivos & 3 & 13,3 \\
\hline
\end{tabular}

Fonte: Relatório de análise textual prcessado pelo programa Iramuteq.

Essa dissociação de teoria e prática e as dificuldades nas habilidades, além de outros aspectos relacionados à vivência dos alunos durante a pesquisa, manifestaram-se na análise de conteúdo realizada com as classes obtidas por meio do processamento do programa Iramuteq e serão discutidas a seguir.

\section{Classe 1: Percepção sobre a oferta de conteúdos oftalmológicos no curso de Medicina}

Essa categoria emerge na fala dos discentes envolvidos na pesquisa e repercute a constatação de artigos recentes da
Tabela 3. Classe 4: Percepção da importância do exame de fundo de olho em pacientes com HAS e DM. Palavras e suas frequências por ordem decrescente das que possuem maior associação com a classe, de acordo $\operatorname{com} X^{2}>3,80, p<0,05$.

\begin{tabular}{ccc}
\hline Palavra & $\boldsymbol{f}$ & $\mathbf{X}^{\mathbf{2}}$ \\
\hline Hipertensão & 8 & 24,1 \\
Diabetes & 8 & 24,2 \\
Quando & 11 & 21,5 \\
Patologia & 8 & 17,8 \\
Precisar & 7 & 16,8 \\
Mostrar & 4 & 11,8 \\
Acreditar & 8 & 11,6 \\
Pontos fortes & 9 & 10,2 \\
Parte prática & 5 & 9,6 \\
Crer & 4 & 8,6 \\
\hline
\end{tabular}

Fonte: Relatório de análise textual processado pelo programa Iramuteq.

Tabela 4. Classe 5: Importância da prática e proposição de técnica de ensino. Palavras e suas frequências por ordem decrescente das que possuem maior associação com a classe, de acordo com $\mathrm{X}^{2}>3,80, \mathrm{p}<0,05$.

\begin{tabular}{ccc}
\hline Palavra & $\boldsymbol{f}$ & $\mathbf{X}^{\mathbf{2}}$ \\
\hline Depois & 10 & 28,8 \\
Aluno & 12 & 22,9 \\
Um no outro & 5 & 20,9 \\
Testar & 4 & 16,6 \\
Direcionar & 4 & 16,6 \\
Ainda & 6 & 16,1 \\
Projeto & 6 & 16,1 \\
Professor & 9 & 14,7 \\
Conhecimento & 7 & 12,0 \\
Prático & 14 & 11,2 \\
\hline
\end{tabular}

Fonte: Relatório de análise textual processado pelo programa Iramuteq.

literatura médica científica nacional e internacional que apontam um tempo cada vez menor despendido a temas relacionados à oftalmologia no ensino médico ${ }^{16,32}$. As falas dos estudantes apresentadas a seguir fazem referência a essa constatação:

[...] porque é muito escasso o nosso contato com a oftalmologia no geral (E6).

[...] pontos fortes foram justamente preencher essa lacuna que ficou na universidade porque falando no meu caso eu fiz uma fundoscopia somente durante cinco anos de curso [...] (E7) 
Considerando o pouco tempo de instalação do curso de Medicina da Uepa, é necessário que se destaque que o exame de fundo de olho, independentemente da técnica usada, é parte integrante do exame clínico do paciente, em especial em situações clínicas relevantes como em pacientes neurológicos e com DM e HAS. Desse modo, é necessário sublinhar que o ensino do exame de fundo de olho não é, em sua essência, um tema exclusivamente oftalmológico, mas antes uma etapa da avaliação clínica geral ${ }^{12}$.

\section{Classe 3: Percepção da retinografia comparada à fundoscopia direta}

Na literatura médica, há uma indicação clara de que o acesso ao exame de fundo de olho está sendo ampliado através da obtenção de imagens de alta resolução com possibilidades de edição e compartilhamento (retinografias digitais) ${ }^{13-15}$. A oftalmoscopia direta necessita de habilidades motoras que precisam ser constantemente exercitadas. A seguir, algumas falas refletem sobre a retinografia digital:

As retinografias digitais elucidam muito $o$ exame, ampliam o campo de visão e deixa de ser examinador dependente em comparação com a fundoscopia, então você pode visualizar melhor todas as estruturas [E2).

Eu acho que é importante análise das retinografias principalmente porque tem um campo visual maior e melhor e mais detalhado para ver [...] (E5)

[...] o profissional consegue ver com mais acurácia e definição a fotografia em si, você consegue aumentar ou diminuir, ir para um campo ou para outro com mais facilidade, talvez um ponto fraco do método seria a ausência do paciente no momento da análise do exame $E(13)$

Os estudantes foram tácitos em constatar os aspectos positivos da retinografia (precisão, confiabilidade e maior campo verificável de retina), porém o interessante de ambientes autênticos (como o utilizado nesta pesquisa) é verificar que os estudantes também foram capazes de formular conceitos como viabilidade econômica e portabilidade do exame:

[...] o principal ponto negativo da retinografia que ele não vai estar acessível em grande parte da população e em grande parte dos municípios $e$ alguns determinados estados, acaba tendo uma alta dificuldade até por conta do preço [...] (E4)

O profissional médico deve exercer essa atitude crítica no sentido de que se possa melhorar a eficiência dos serviços de saúde; a obtenção de imagens deverá ter seus custos reduzidos e ter portabilidade mas, no momento, as retinografias dependem de máquinas ainda pouco acessíveis. Essa postura crítica é uma das características que se almejam no perfil do egresso dos cursos de Medicina ${ }^{41-44}$.

Classes 4 e 5: Percepção sobre a relevância do exame de fundo de olho em pacientes com DM/HAS, proposição de técnica de ensino e importância da prática

Nas metodologias ativas de aprendizagem, em especial abordagem baseada em problemas, a identificação de um tema relevante que seja vivenciado pelo aluno na realidade e que possa se constituir no que se conceitua "elemento deflagrador "ou "gatilho" é um dos primeiros passos no processo de ensino. A partir desse problema pode-se levar o aluno a definir a dimensão do seu saber prévio e perceber por si mesmo a região de fronteira do seu "não saber"46-49.

Esse momento da pesquisa conduz à necessidade, considerando a taxonomia de Bloom ${ }^{50}$ de objetivos educacionais, de que, no domínio cognitivo, os alunos de Medicina devem entender sinais patológicos do fundo de olho na DM e HAS como o resultado de alterações sistêmicas da arquitetura e funcionalidade vascular que geram os fenômenos isquêmicos e tromboembólicos que caracterizam as complicações desses agravos.

A importância do exame de fundo de olho para pacientes com HAS e DM surge na classe 4 gerada com a análise do Iramuteq.

[...] retinografia digital é um exame excelente, então a gente pode identificar bastante coisa com a retinografia principalmente em populações-alvo, pacientes idosos, hipertensos, diabéticos (E11).

[...] incrementar a quantidade de aula sobre fundoscopia e fazer esta correlação com outros módulos para a gente entender que a fundoscopia não é algo localizado, mas que também envolve outros sistemas [...] E10.

[...] três pontos fortes que posso citar é primeiro teve uma visão geral de todo o processo que pode ocasionar estas alterações na retina, todo o processo da patologia crônica da hipertensão e do diabetes [...] (E6)

Verifica-se que os estudantes expressaram conceitos como vulnerabilidade e análise sistêmica, fundamentais no campo do exercício da clínica médica. Prática e teoria são componentes de ensino que necessitam constantemente se fundir. Os discentes estão preocupados com "a prática", entendem que o saber se efetua na relação da esfera do conhecimento com a realidade concreta e objetiva. $O$ termo "prática" é uma constante que surge nas classes 4 e 5 :

Acredito que o exame do fundo de olho tem que ter a prática, não adianta ficar só na teoria porque a gente aprende verdadeiramente na prática (E2).

[...] em relação ao que eu me senti mais interessada, foi 
realmente com a questão da prática (E21)

[...] nós tivemos tanto a teoria que embasou a nossa prática, mas o maior interesse, se fosse para destacar um ou outro, seria a parte prática (E12).

$\mathrm{Na}$ oftalmoscopia direta a aproximação com o paciente requer nível considerável de habilidade manual; nas observações de campo nenhum dos estudantes possuía treino dessas habilidades e todos necessitaram de sucessivas tentativas ou abordagens para que pudessem localizar o ponto de referência do disco ótico e da emergência dos vasos da retina. Nas observações de campo foram frequentes anotações condizentes com níveis de ansiedade e frustração nas tentativas iniciais sem êxito assim como as de euforia nos resultados positivos. Atitudes emocionais nem sempre podem ser trabalhadas em ambientes de simulação, visto que os estímulos vindos da realidade e os virtuais não são equivalentes.

Para contornar essas dificuldades, os grupos de discentes foram unânimes em propor, durante os desenvolvimentos da pesquisa, o treinamento entre pares, o que foi consentido. Essa proposição ativa e as dificuldades no processo de ensino e aprendizagem se explicitam na classe 5 e falas correspondentes são resgatadas a seguir:

[...] na prática como segurar o aparelho a ver as dificuldades de visualização e de localização do nervo ótico, de como se portar diante do paciente, o limite de distância entre você e o paciente na hora de fazer o exame, e o incômodo que a luz pode trazer para o olho do paciente, ele pode acabar piscando também durante o momento (E2)

[...] porque a gente com o mínimo de treinamento hoje, na primeira vez a gente já conseguiu ter um acesso, ver realmente o nervo ótico e as estruturas, é questão de prática (E6).

Trabalhos da literatura médica não exploram com propriedade o ensino das habilidades na oftalmoscopia. $O$ exame clínico bem conduzido repetidas vezes é de extrema importância na formação médica para que se fixem os parâmetros e aspectos normais do organismo humano e se reconheçam sinais e sintomas que deles se diferenciam e que constituem a base dos diagnósticos sindrômicos.

Esta pesquisa envolveu uma população pequena de estudantes, assim como possui limitações pelo fato de não ter utilizado instrumentos padronizados para observações de campo. Este estudo permitiu, porém, verificações importantes como a dissociação entre teoria e prática sobre o ensino do exame de fundo de olho e o potencial pedagógico (mesmo consideradas suas limitações) da oftalmoscopia direta.

\section{CONCLUSÕES}

Técnicas reprodutíveis para avaliação do fundo de olho ancoradas em conceitos de aprendizagem significativa com adequada integração de teoria e prática podem formar um conjunto útil para otimizar a abordagem desse tema no ensino médico, que deve estar em consonância com as novas tecnologias disponíveis na área da saúde. Assim, é plausível que a obtenção e análise de imagens de fundo de olho sejam consideradas como objetivos da educação médica. É importante que o estudante de Medicina compreenda de forma abrangente a relevância da análise do fundo de olho nos ambientes e nas situações que demandem essa avaliação. A eficiência na técnica de oftalmoscopia direta requer um treino contínuo de habilidades e ainda pode ocupar um espaço na educação médica e na prática clínica. O ensino do exame do fundo de olho deve ocorrer idealmente de maneira integrada e contínua às experiências reais vivenciadas pelo estudante. Reprodutibilidade técnica, ensino contextualizado e integração de teoria e prática podem ser pontos-chave para que o médico em formação supere dificuldades e mantenha competências para o exame do fundo de olho no contexto epidemiológico de sua atuação.

\section{CONTRIBUIÇÃO DOS AUTORES}

Norimar Pinto de Oliveira supervisionou a pesquisa. Robson Jose de Souza Domingues participou da supervisão e avaliação do artigo. Edmundo Frota Almeida de Almeida Sobrinho perticipou da supervisão técnica do artigo. Simone Argentino participou da revisão do artigo. Ellen Ágatta Marinho Silva participou do processamento de dados com o uso do programa Iramuteq. Marcelo Dias Ferreira Júnior transcreveu as entrevistas.

\section{CONFLITO DE INTERESSES}

Declaramos não haver conflito de interesses.

\section{FINANCIAMENTO}

Declaramos não haver financiamento.

\section{REFERÊNCIAS}

1. Ivan $Y$, Ramgopal S, Cardenas-Villa M, Winger DG, Wang L, Vitale MA, et al. Feasibility of the digital retinography system camera in the pediatric emergency department. Pediatr Emerg Care. 2018;34(7):488-91 [acesso em 29 dez 2019]. Disponível em: https://pubmed.ncbi.nlm.nih. gov/28609333/.

2. Kouzmitcheva E, Grover SA, Berenbaun T, Ali A, Atikson A, Yeh EA Evaluation of an ophthalmoscopy simulator to teach funduscopy skills to pediatric residents. Can J Neurol Sci. 2018;45(3);320-4 [acesso em $27 \mathrm{dez}$ 2019]. Disponível em: https://pubmed.ncbi.nlm.nih.gov/29455676/.

3. Benbassat J, Polak BCP, Javitt JC. Objectives of teaching direct ophthalmoscopy to medical students. Acta Ophthalmol. 2011;90(6):5037 [acesso em 29 dez 2019]. Disponível em: https://pubmed.ncbi.nlm.nih gov/22040169/. 
4. Gurney SP, Makanjuola T, Kutubi M, Parulekar M, Abbott J. How to use the direct ophthalmoscope. Arch Dis Child. 2018;103(2):102-9 [acesso em 29 dez 2019]. Disponível em: https://pubmed.ncbi.nlm.nih.gov/28935833/.

5. Lima MA, Maranhão-Filho P. O que é essencial no exame neurológico. Arq Neuro-Psiquiatr. 2012; 70(12):939-41: doi:10.1590/S0004-282X2012001200007.

6. Mackay DD, Bruce BB. Nom-mydriatic fundus photography: a practical review for the neurologist. Pract Neurol. 2016;16(5):543-51 [acesso em 29 dez 2019]. Disponível em: https://pubmed.ncbi.nlm.nih.gov/27407177/.

7. Rodenbeck SJ, Mackay DD. Examining the ocular fundus in neurology. Curr Opin Neurol. 2019; 2(1):105-10 [acesso em 29 dez 2019]. Disponível em: https://journals.lww.com/co-neurology/Abstract/2019/02000/Examining the_ocular_fundus_in_neurology.18.aspx.

8. Schulz C, Moore J, Hassan D, Tamsett E, Smith CF. Adressing the "forgotten art of fundoscopy": evaluation of a novel teaching oftalmoscope. Eye (Lond). 2016;30(3):375-84 [acesso em 29 dez 2019]. Disponível em: https:// pubmed.ncbi.nlm.nih.gov/26563657/.

9. Schulz C, Hodgkins P. Factors associated with confidence in fundoscopy. Clin Teach. 2014;11(4):431-5 [acesso em 29 dez 2019]. Disponível em https://pubmed.ncbi.nlm.nih.gov/25212928/.

10. Yusuf $\mathrm{IH}$, Patel CK. A oftalmoscopia direta deve ser ensinada a estudantes de medicina - Sim. Eye (Lond). 2015;29(8):987-9 [acesso em 29 dez 2019]. Disponível em: https://por.kyhistotechs.com/direct-ophthalmoscopyshould-be-taught-undergraduate-medical-studentsyes-88680464.

11. Purbrick RMJ, Chong NV. A oftalmoscopia direta deve ser ensinada a estudantes de medicina - Não. Eye (Lond). 2015;29(8):990-1 [acesso em 29 dez 2019]. Disponível em: https://por.kyhistotechs.com/ direct-ophthalmoscopy-should-be-taught-undergraduate-medicalstudentsyes-88680464.

12. Kahwage Neto SG, Braga TKK, Portella MB, Andriolo RB. Ensino de habilidades clínicas e a aplicabilidade de um guia simplificado de exame físico na graduação de Medicina. Rev Bras Educ Med. 2017;41(2):299-309 [acesso em $26 \mathrm{dez}$ 2019]. Disponível em: https://www.scielo.br/scielo. php?pid=S010055022017000200299\&script=sci_abstract\&tlng $=$. .

13. Biousse V, Bruce BB, Newman NJ. Ophtalmoscopy in the 21st century: the 2017. H. Houstons Merrit Lecture. Neurology. 2018;90(4):167-75 [acesso em 29 dez 2019]. Disponível em: https://pubmed.ncbi.nlm.nih. gov/29273687/.

14. Gunassekera CD, Thomas P. High-resolution direct ophthalmoscopy with an unmodified iPhone X JAMA. Ophthalmol. 2019;137(2):212-3 [acesso em 29 dez 2019]. Disponível em: https://pubmed.ncbi.nlm.nih. gov/30489602/.

15. Bruce BB, BiousseV, Newman NJ. Nonmydiatric ocular fundus photography in neurologic emergencies. JAMA Neurol. 2015;72(4):455-9 [acesso em 29 dez 2019]. Disponível em: https://pubmed.ncbi.nlm.nih.gov/25665183/.

16. Mantora S, Sandinha MT, Ajith A, Song A, Steel DHW. Smart phone ophthalmoscopy: a potential replacement for the direct ophthalmoscope. Eye (Lond). 2018;32(11):1766-71 [acesso em 29 dez 2019]. Disponível em: https://pubmed.ncbi.nlm.nih.gov/30042410/.

17. Wu AR, Fouzdar-Jain S, Suh DW. Comparison Study of funduscopic examination using a smartphone-based digital ophthalmoscope and the direct ophthalmoscope. J Pediatr Ophthalmol Strabismus. 2018;55(3):2016 [acesso em $29 \mathrm{dez} 2019]$ ]. Disponível em: https://europepmc.org/article/ $\mathrm{med} / 29796680$.

18. Ah-Kee EY, Khan AA, Lim LT. Ocular fundus photography: an alternative to ophthalmoscopy for teaching. Med Teach. 2016;38(5):529-30 [acesso em 29 dez 2019]. Disponível em: https://pubmed.ncbi.nlm.nih.gov/26372019/.

19. Bifolck $E$, Fink A, Pederesen $D$, Gregory T. Smartphone imaging for the ophthalmic examination in primary care. JAAPA. 2018;31(8):34-8 [acesso em 28 dez 2019]. Disponível em: https://pubmed.ncbi.nlm.nih. gov/30048353.

20. Bruce BB. Nonmydiatric ocular fundus photography in the emergency department: how it can benefit neurologists. Semin Neurol. 2015;35(5):4915 [acesso em 29 dez 2019]. Disponível em: https://pubmed.ncbi.nlm.nih. gov/26444394/
21. Omer MT, Abbas E. Opticard: an inexpensive and portable method of bedside direct fundoscopy. J Coll Physicians Surg Pak. 2017;27(11):719-21 [acesso em $29 \mathrm{dez}$ 2019]. Disponível em: https://pubmed.ncbi.nlm.nih. gov/29132486/.

22. Alves AP, Santos RWV, Sobrinho EFA, Rocha SPL, Loch ACN. Retinopatia em pacientes diabéticos e/ou hipertensos em uma unidade de saúde da família. Rev Bras Oftalmol. 2014;73(2):108-11 [acesso em 29 dez 2019]. Disponível em: https://www.scielo.br/pdf/rbof/v73n2/0034-7280rbof-73-02-0108.pdf.

23 Brasil. Cadernos de atenção básica. Estratégias para o cuidado da pessoa com doença crônica - diabetes mellitus. Diário Oficial da União, Brasília; 15 dez 2013. Seção 1, p. 30 [acesso em 29 dez 2019]. Disponível em: https:// bvsms.saude.gov.br/bvs/publicacoes/estrategias_cuidado_pessoa_ diabetes_mellitus_cab36.pdf.

24. Brasil. Cadernos de atenção básica. Cuidados da pessoa com doença crônica - hipertensão arterial sistêmica. Diário Oficial da União, Brasília 10 set 2013. Seção 1, p. 28 [acesso em 29 dez 2019].Disponível em: https:// bvsms.saude.gov.br/bvs/publicacoes/estrategias_cuidado_pessoa_ doenca_cronica_cab35.pdf.

25. Sociedade Brasileira de Diabetes. Diretrizes da Sociedade Brasileira de Diabetes. São Paulo: Clannad, 2017 [acesso em 29 dez 2019]. Disponível em: https://www.diabetes.org.br/profissionais/images/20 17/diretrizes/ diretrizes-sbd-2017-2018.pdf.

26. Akaishi Y, Otaki J, Takahashi O, Breugelmans R, Kojima K, Seki M, et al. Validity of direct ophthalmoscopy skill evaluation with ocular fundus examination simulators. Can J Ophthalmol. 2017;49(4):377-81 [acesso em 26 dez 2019]. Disponível em: https://pubmed.ncbi.nlm.nih.gov/25103656/

27. Ricci $\mathrm{LH}$, Ferraz CA. Modelos de simulação aplicados ao ensino prático e aprimoramento de habilidades em oftalmoscopia direta e indireta: uma revisão. Arq Bras Oftalmol. 2014;77(5):334-8 [acesso em 29 dez 2019]. Disponível em: https://www.scielo.br/pdf/abo/v77n5/0004-2749abo-77-05-0334.pdf.

28. Yusuf IH, Ridyard E, Fung THM, Sipkowa Z, Patel CK. Integrating retinal simulation with a peer-assessed group OSCE format to teach direct ophthalmoscopy. Can J Ophthalmol. 2017;52(4):392-7 [acesso em 29 dez 2019]. Disponível em: https://pubmed.ncbi.nlm.nih.gov/28774522/.

29. Yusuf $\mathrm{IH}$, Yank E, Knigt K, Leaver L. Direct ophthalmoscopy: teaching in primary care. Clin Teach. 2016;13(3):235-7 [acesso em 29 dez 2019]. Disponível em: https://pubmed.ncbi.nlm.nih.gov/25982427/.

30. Bernard SE, Kwok J, Liao W, Baxter S. Use of a fundus photograph matching program in imparting proficiency in ophthalmoscopy Can J Ophthamol. 2017;53(5):480-5 [acesso em 29 dez 2019]. Disponível em: https://pubmed.ncbi.nlm.nih.gov/30340715/.

31. Kelly LP, Garza PS, Bruce BB, Graubart EB, Newman NJ, Biousse V. Teaching ophthalmoscopy to medical students (the TOTeMS study). Am J Ophthalmol. 2013;156(5):1056-61 [acesso em 29 dez 2019]. Disponível em: https://pubmed.ncbi.nlm.nih.gov/24041982/.

32. Martin TG, Costa ALF, Helene O, Martins RV, Helene AF, Schor P. Training of direct ophthasmocopy using models. Clin Teach. 2017;14(6):423-6 [acesso em 27 dez 2019]. Disponível em: https://pubmed.ncbi.nlm.nih. gov/2840173.

33. Borgersen NJ, Skou TAS, Konge L, Serensen TL, Subhi Y. Virtual realitybased proficiency test in direct ophthalmoscopy. Acta Ophthalmol. 2018:96(2):59-61 [acesso em 29 dez 2019]. Disponível em: https:// pubmed.ncbi.nlm.nih.gov/28834332/.

34. Mackay DD, Garza PS. Ocular fundus photography as an educational tool. Semin Neurol. 2015; 35(15):496-505 [acesso em 29 dez 2019]. Disponível em: https://www.thieme-connect.com/products/ejournals/ abstract $/ 10.1055 / \mathrm{s}-0035-1563572$ ?device $=$ mobile

35. Kwok J, Liao W, Baxter S. Evaluation of an online peer fundus photograph matching program in teaching direct ophthalmoscopy to medical students. Can Ophthalmol. 2017;52(5):441-6 [acesso em 29 dez 2019]. Disponível em: https://pubmed.ncbi.nlm.nih.gov/28985801/.

36. Chamon W, Schor P. Ensinando oftalmologia aos estudantes de medicina: uma nova abordagem. Arq Bras Oftalmol. 2012;75(1):5-7 [acesso em 29 dez 2019]. Disponível em: https://www.scielo.br/scielo.php?pid=S0004$27492012000100001 \&$ script=sci_arttext. 
37. Kulkarni S, Seneviratne N, Baig MS, Khan AHA. Artificial intelligence in medicine: where are we now? Acad Radiol. 2020;27(1):62-70 [acesso em 28 dez 2019]. Disponível em: https://pubmed.ncbi.nlm.nih.gov/31636002

38. Van der Heijden AA, Abramoff MD, Verbraak F, Van Hecke MV, Liem A, Nijpels G. Validation of automated screening for referable diabetic retinopathy with the IDx-DR device in the Hoorn Diabetes Care System. Acta Ophthalmol. 2018;96(1):63-8 [acesso em 28 dez 2019]. Disponível em: https://pubmed.ncbi.nlm.nih.gov/29178249/.

39. Rajala R, Subashini R, Anjana RM, Mohan V. Automated detection of diabetic 27 retinopathy in smartphone photography using artificial intelligence. Eye (Lond). 2018;32(6):1138-44 [acesso em 29 dez 2019]. Disponível em: https://pubmed.ncbi.nlm.nih.gov/29520050/.

40. Divya K, Suvetha K, Sen A, Sundar D. Needs assessment of ophthalmology education for undergraduate medical students - a study from a medical college in South India. Educ Health (Abingdon). 2017;30(3):223-7 [acesso em 29 dez 2019]. Disponível em: https://pubmed.ncbi.nlm.nih. gov/29786024/.

41. Brasil. Resolução CNE/CES n 3, de 20 de junho de 2014. Institui Diretrizes Curriculares Nacionais do Curso de Graduação em Medicina. Diário Oficial da União, Brasília; 20 jun 2014. Seção 1, p. 30 [acesso em 29 dez 2019]. Disponível em: http://portal.mec.gov.br/escola-de-gestoresda-educacao-basica/323-secretarias-112877938/orgaos-vinculados82187207/20138-ces-2014.

42. Ellery AEL, Bossi MLM, Loiola FA. Integração ensino, pesquisa e serviços em saúde: antecedentes, estratégias e inciativas. Saúde Soc São Paulo. 2013;22(1):187-98 [acesso em 29 dez 2019]. Disponível em: https://www. scielo.br/pdf/sausoc/v22n1/17.pdf.

43. Farias AMP, Martin ALAR, Cristo CS. Aprendizagem ativa na educação em saúde: percurso histórico e aplicações. Rev Bras Educ Med. 2015;39(1):14858 [acesso em 29 dez 2019]. Disponível em:https://www.scielo.br/scielo. php?pid=S0100-55022015000100143\&script=sci_abstract\&tlng=pt

44. Vendruscolo C, Prado ML, Kleba ME. Integração ensino-serviço no âmbito do programa nacional de reorientação da formação profissional em saúde. Ciênc Saúde Colet. 2016;21(9):2949-60 [ac,esso em 29 dez 2019]. Disponível em: https://www.scielo.br/scielo.php?script=sci abstract\&pid=S141381232016000902949\&lng=en\&nrm=iso\&tlng=pt.
45. Meireles MAC, Fernandes CP, Silva LS. Novas diretrizes curriculares nacionais e a formação médica: expectativas dos discentes do primeiro ano do curso de Medicina de uma instituição de ensino superior. Rev Bras Educ Med. 2019;43(2):67-78 [acesso em 27 dez 2019]. Disponível em:https://www.scielo.br/scielo.php?script=sci abstract\&pid=S010055022019000200067\&lng=en\&nrm=iso\&tlng=p.

46. Panúncio-Pinto MP, Troncon LEA. Avaliação do estudante: aspectos gerais Medicina (Ribeirão Preto). 2014;47(3):314-23 [acesso em 29 dez 2019]; 314-23. Disponível em: http://revista.fmrp.usp.br/2014/vol47n3/10_ valiacao-do-estudante-aspectos-gerais.pdf.

47. Prestes EX, Palacios VRCM, Ohnishi MDO, Portella MB, Brito MVH Barreto BAP. Projeto Político Pedagógico do Curso de Medicina da Universidade do Estado do Pará (UEPA). Belém: Uepa; 2015 [acesso em 29 dez 2019]. Disponível em: https://webcache.googleusercontent.com/ search?q=cache:7GMdONk_yo0J:https://paginas.uepa.br/ccbs/edfisica/ files/PPP_UEPA.pdf $+\& c d=2 \& h l=p t-B R \& c t=c l n k \& g l=b r$.

48. Leon LB, Onofrio FQ. Aprendizagem baseada em problemas na graduação médica: uma revisão da literatura atual. Rev Bras Educ Med. 2015;19(4):6149 [acesso em 29 dez 2019]. Disponível em: https://www.scielo.br/scielo. php?pid=S0100-55022015000400614\&script=sci_abstract\&tlng=pt.

49. Telang A. Problem-based learning in health professions education: an overview. Archives of Medicine and Health Sciences. 2014:2(2):243-6 [acesso em 29 dez 2019]. Disponível: http://www.amhsjournal.org/text. asp?2014/2/2/243/144363.

50. Oliveira APSB, Pontes JNA, Marques MA. O uso da taxonomia de Bloom no contexto da avaliação por competência. Pleiade. 2016;10(20):12-22 [acesso em 29 dez 2019]. Disponível: https://pleiade.uniamerica.br/index. $\mathrm{php} /$ pleiade/article/view/306. 